\title{
LA-UR-18-25921
}

Approved for public release; distribution is unlimited.

Title: $\quad$ Probability of Encounter between Ports of Entry (PrEP): Project Final Report

Author(s): $\quad$ Ambrosiano, John Joseph

Dauelsberg, Lori Rose

Tompkins, George

Intended for: Report

Issued: 
Disclaimer:

Los Alamos National Laboratory, an affirmative action/equal opportunity employer, is operated by the Los Alamos National Security, LLC for the National Nuclear Security Administration of the U.S. Department of Energy under contract DE-AC52-06NA25396. By approving this article, the publisher recognizes that the U.S. Government retains nonexclusive, royalty-free license to publish or reproduce the published form of this contribution, or to allow others to do so, for U.S. Government purposes. Los Alamos National Laboratory requests that the publisher identify this article as work performed under the auspices of the U.S. Department of Energy. Los Alamos National Laboratory strongly supports academic freedom and a researcher's right to publish; as an institution, however, the Laboratory does not endorse the viewpoint of a publication or guarantee its technical correctness. 


\section{Probability of Encounter between Ports of Entry (PrEP): Project Final Report}

John Ambrosiano, Lori Dauelsberg, George Tompkins

July 2, 2018

\section{Overview}

To enhance detection and interdiction of radiological or nuclear material trafficking between primary POEs into the US, The Department of Homeland Security is faced with highly variable smuggling pathways through expansive geographic regions. The goal of this project is to develop an approach to estimate likely pathways, as well as the probability of encounter along any given path.

Our aim has been to design and test a novel approach based on assessing the adversary's perception of discovery likelihood over a broad geographic region, and using that result to estimate an "aversion force field" over the same region. The aversion force field is derived from analogies based on the physics of electric charges. Combining the aversion force with the intention of an adversary to reach their destination, generates a route that is consistent with both the adversary's intention and their aversion to deterrent measures.

The project as originally planned had four phases organized as a spiral development plan:

1. Feasibility demonstration and review - The aim of this phase was to explore the novel concept introduced by this project and to demonstrate its feasibility. Specifically it involved implementing the principle model components: (1) Bayesian network model of an adversary's perception of discovery likelihood; and (2) physics-inspired aversion field model; and (3) agent based model of adversary movement across the region of interest. Preliminary versions of these components would be tacitly integrated and then tested on a model configuration as a feasibility demonstration before moving on to other phases.

2. Component model integration and preliminary simulations - The second phase was intended as the next spiral in development, enhancing and refining the code developed in first phase, and to be followed in the third phase by exacting critical tests of the model's components, both separately and together.

3. Full model completion and critical simulations - In the third phase, a fully functioning model would be assembled and challenged by a set of critical test simulations. The version of the code resulting from this phase would thus be considered ready for application to mission-critical problems within the overall DHS modeling architecture.

4. Integration of the full model with DHS modeling architecture - Following completion of critical tests in the prior phase, the PrEP model, in Phase 4, would be available as a stand-alone application, and ready for integration within a larger modeling and simulation architecture. As originally planned, this would include integration with force-on-force-style simulations using frameworks such as DTRA's FREAK, as well as in collaboration with models for pathway prediction and analysis like PEM/Patriot, as well as with detection resource optimization models like NINESIM through the SWORD framework. 
Figure 1 illustrates the intended role of PrEP within the larger DHS architecture for evaluating and countering the threat of smuggling radiological/nuclear materials. As a stand-alone capability, PrEP would be able to evaluate the likelihood that an adversary choosing to travel over open territory would be found at a given location in the region of interest. The capability could also be used in collaboration with tools such as PEM/Patriot combining statistical route distributions over open territory with discrete routes based on conventional road use. Whether stand-alone or integrated with a conventional pathway model, PrEP would provide estimates of the probability of encountering an adversary at some particular location in the region of interest. Such estimates can then be used in conjunction with NINESIM for optimizing detection probability, or for deploying defending forces or other countermeasures.

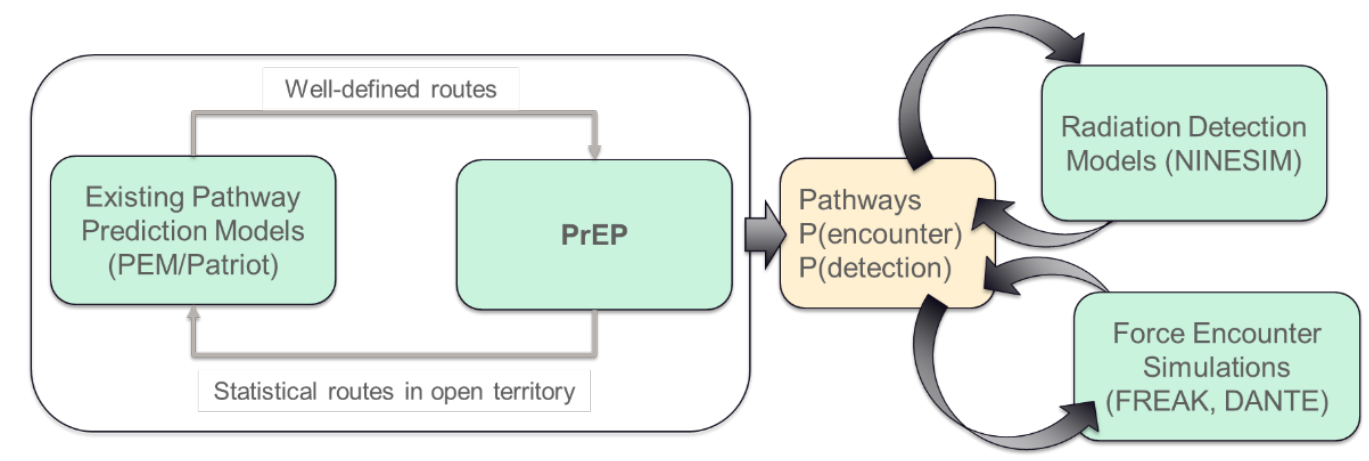

Figure 1. An illustration of PrEP's intended role in the larger DHS architecture of modeling capabilities.

After a successful feasibility review at the completion of Phase 1, changes in mission priorities at DHS led to a discontinuation of the project. As a result, this report will document only Phase 1.

\section{The Bayesian Aversion Perception Model}

The initial Bayesian network developed in Phase 1 assumes infiltration on foot by a sophisticated adversary whose aim is to reach their destination without being observed. It is based on U.S. Army Field Manual FM 7-93. ${ }^{1}$ The doctrine described in the manual holds that infiltrators will prefer surroundings that make them hard to observe and unlikely to be encountered.

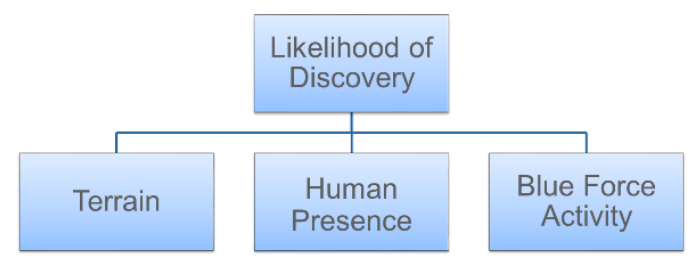

Figure 2. The high-level features of the Bayesian risk aversion model component.

Based on this principle, a preliminary Bayesian model was developed that requires no initial subject matter input, relying only on geographic terrain and population features. The general structure of the model is shown in Figure 2 which states that the general factors contributing to the adversary's perception of discovery likelihood at any point in the region are (1) the terrain and other features of the landscape; (2) the presence of anyone that could observe the adversary and potentially report them; and (3) the presence of any defending forces.

\footnotetext{
${ }^{1}$ United States Army Field Manual 7-93 Long-Range Surveillance Unit Operations.
} 
As these factors are refined, we obtain the model structure shown in Figure 3. Under terrain, the factors include the slope, roughness, and type of ground cover. Human presence is represented as population density, and Blue Force (defensive) activity is linked to the distance at any location to the nearest fence or other barrier. These factors are not exclusive. Rather, they were chosen because they are representative of factors in that category, are expected to have a substantial effect on the adversary, and are suitable for demonstrating feasibility. We believe that the factors in the figure represent a good general structure for any adversary risk aversion model.

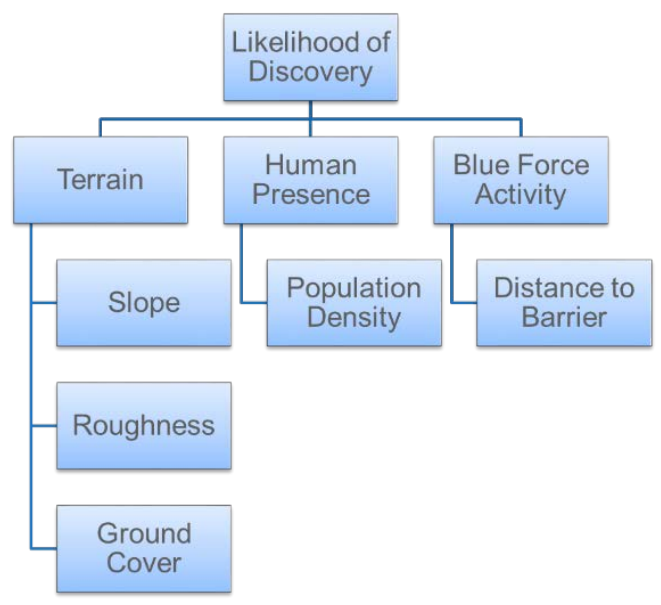

Figure 3. The next level of refinement in the Bayesian risk aversion model. Here specific factors related to terrain, human presence, and Blue Force activity have been expanded upon.

We expect different adversary types to perceive risk differently. However, we expect all human adversaries with similar goals (i.e., of avoiding discovery) to react in various ways to the same factor. Therefore, having a good general structure for the aversion model is important since it allows us to accommodate different adversary types by adjusting the model (adjusting probabilities, adding specific influences to more general ones, and so on), rather than inventing entirely new models.

Based on these factors a Bayesian network was created using plausible values for the conditional probabilities. A diagram of the model, as displayed in the Netica Bayesian network modeling tool, is shown in Figure 4. For the feasibility demonstration, we assumed a uniform, low-level population density for the sparsely-populated test region, rather than apply a detailed geographic population layer.

The test region in question is shown in Figure 5. This region was chosen because of its limited extent, and also because it includes rugged and smooth terrain, as well as open and obscuring ground cover. The region also contains several sections of a border fence.

Using this test region, each of the prior factors in the Bayesian model were evaluated at cells of a geographic grid. These inputs grids were then provided to GeoNetica, a geographically aware version of the Netica tool that can evaluate the same model at each cell of a geographic grid based on the input data assigned to the same cell.

The input grids are shown in Figure 6. They are (a) percent slope, (b) roughness, (c) land cover category, and (d) proximity to a border fence. 


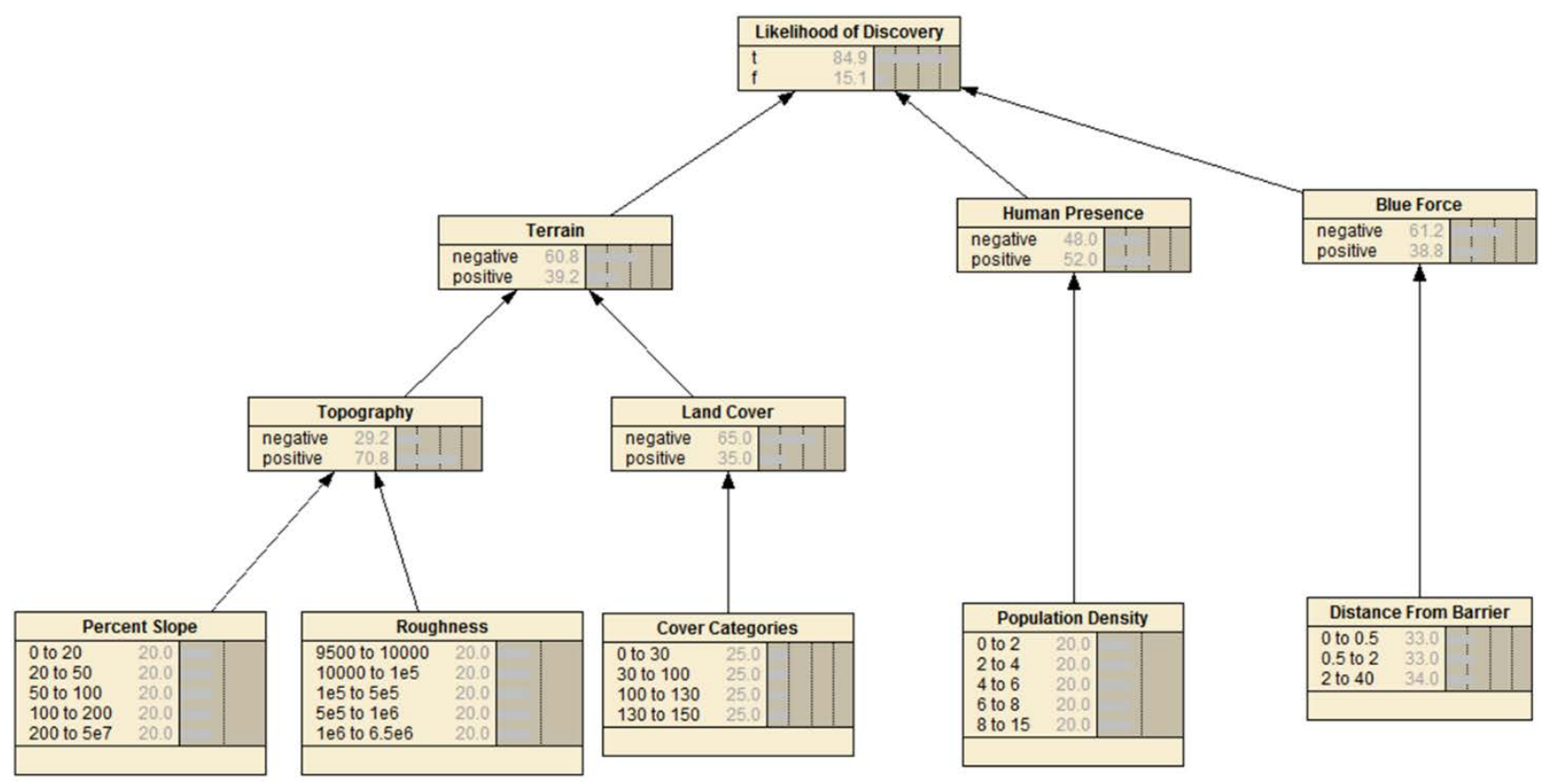

Figure 4. A Bayesian network model of perceived discovery likelihood based on US Army Field Manual 7-93.

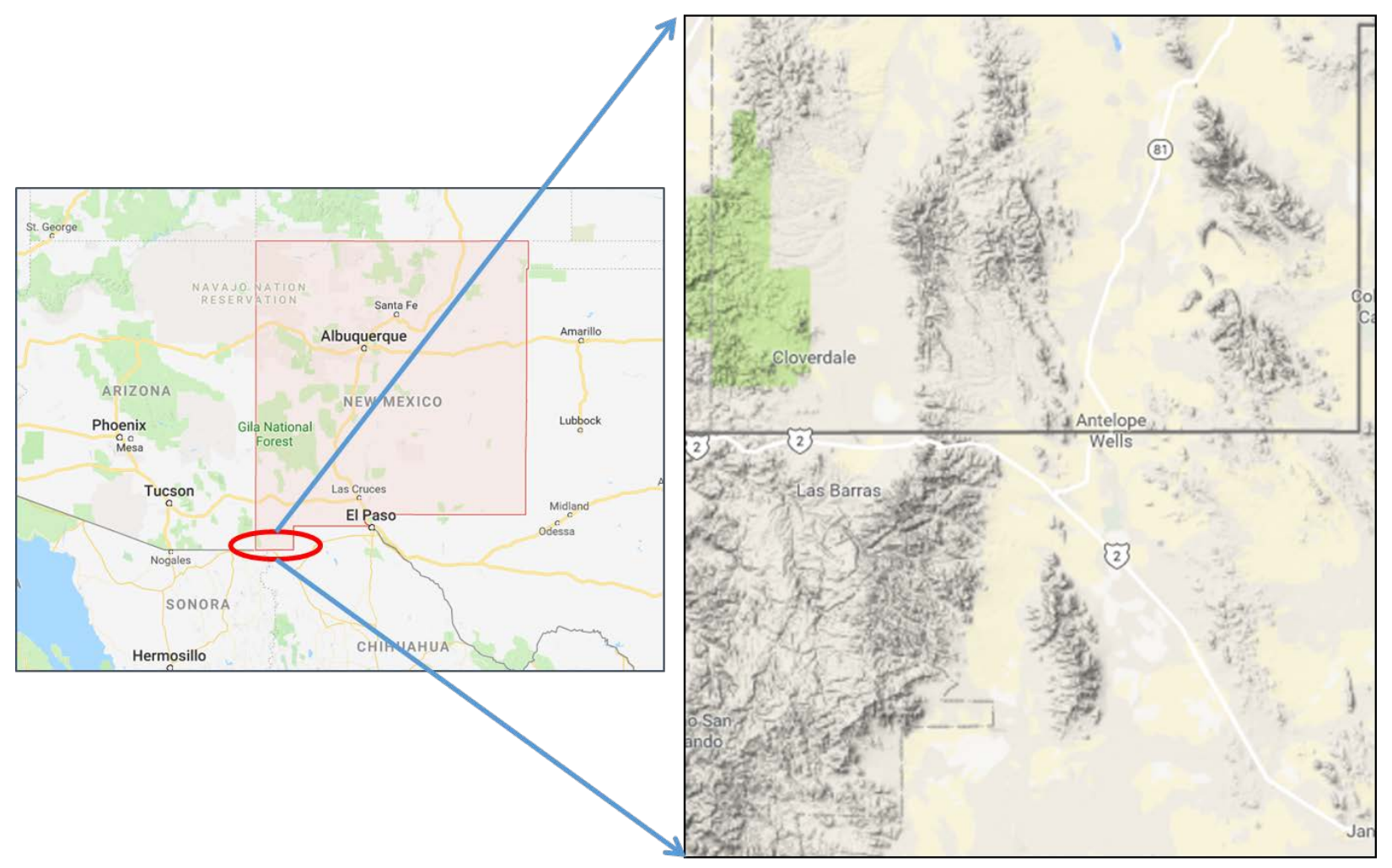

Figure 5. The test region for the feasibility demonstration. 

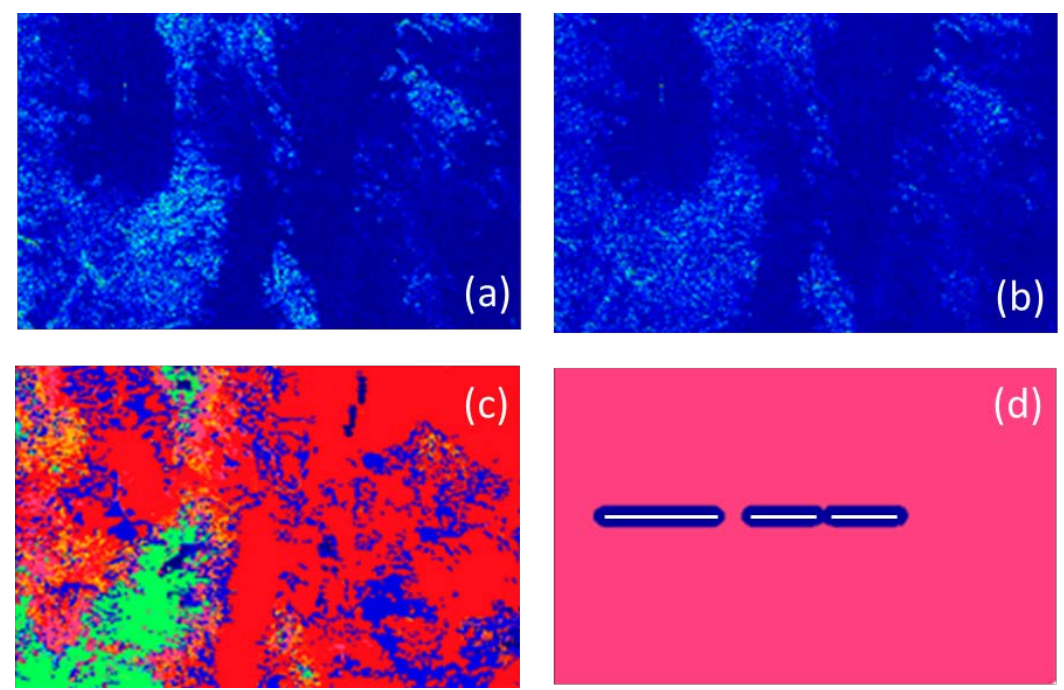

Figure 6. The input grids for the Bayesian network: (a) percent slope, (b) roughness, (c) land cover category, and (d) proximity to a border fence.

The output of the Bayesian network based on these inputs is shown in Figure 7, where we have superimposed the results on a terrain map of the region.

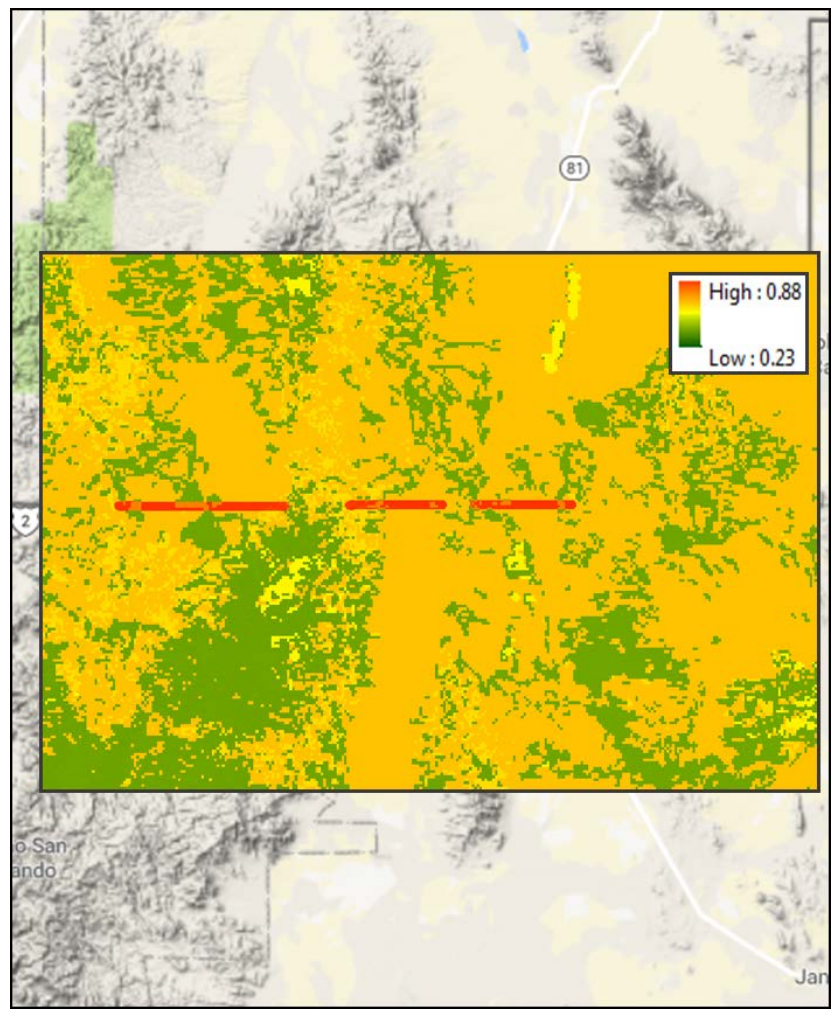

Figure 7. The output of the Bayesian network model (probability of discovery) over the geographic test region.

One can see from the figure that the likelihood values range from nearly $90 \%$ in immediate vicinity of the border fence, to less than $25 \%$ where the terrain is favorable to the adversary remaining unobserved. Open territory is perceived as relatively risky, that is, around $65 \%$. 
For comparison, we have modified the Bayesian model probabilities to represent a second type of adversary. This adversary is similar to the first except that they have a somewhat lower sense of risk in open territory or in proximity to a fence. That is, they are not quite as averse to discovery as the first infiltrator. The results of that model are shown in Figure 8 using the same scale for the color map.

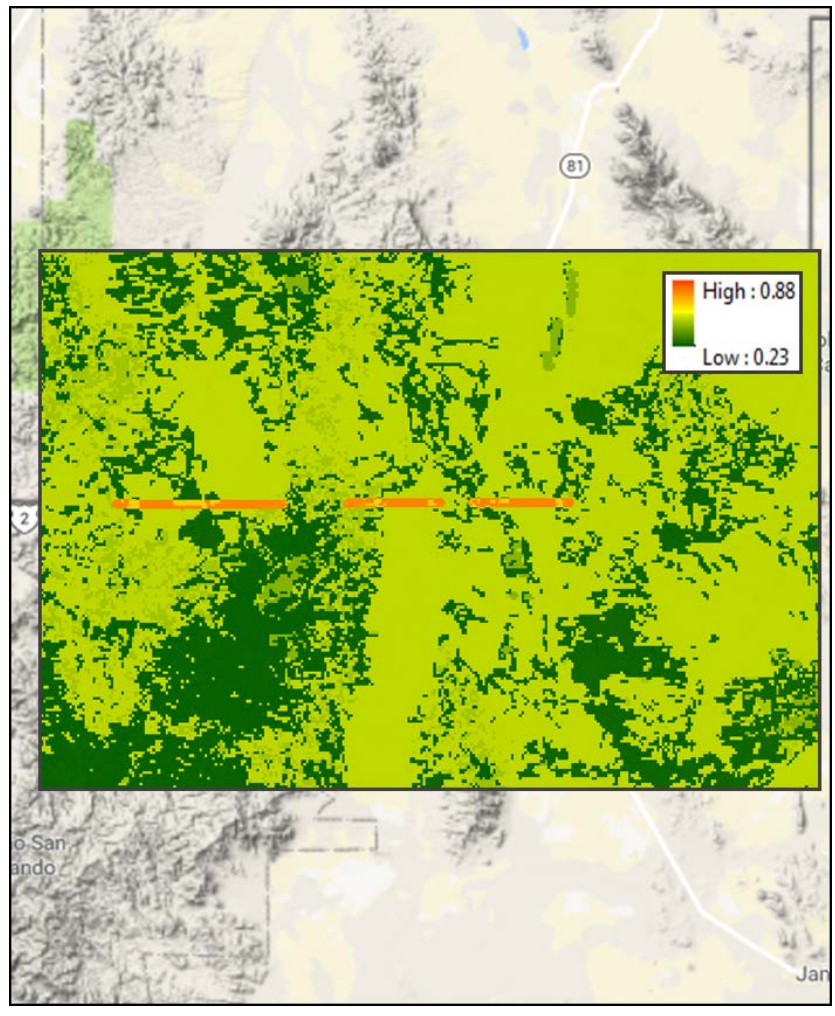

Figure 8. Output of the Bayesian network for a second type of infiltrator with somewhat more tolerance for open territory.

The results are consistent with the second type of infiltrator being less concerned with exposure in open territory (likelihood perceived as $\sim 50 \%$ ). Both types of infiltrator find close proximity to the fence highly threatening. We have developed a model that describes an adversary's perceived likelihood of discovery/detection

In summary, we have demonstrated that a Bayesian network model of adversary aversion to discovery, based on a credible description of the factors driving an infiltrator on foot, produces plausible results.

In demonstrating the model, we have used actual geographic data for a portion of the U.S.-Mexican border. Results such as those demonstrated here are input to the next stage of the modeling process.

Some things to note regarding the Bayesian network demonstration:

- The current model is hypothetical

- The model has not been reviewed by subject matter experts (e.g., CBP personnel)

- It has not yet been calibrated with actual border crossing/apprehension data 


\section{The Aversion Force Field Model}

The aversion force-field is a repulsive force that causes adversaries to deviate from their intended route. The model draws an analogy between the repulsion of electric charges, and features in a geographic landscape to which an adversary is averse.

The source of the force field is dependent on the perceived discovery likelihood calculated by the Bayesian network component. What follows is a brief description of how this result becomes an aversion force field.

\section{The Electric Fields Analogy for the Aversion Force}

Every charged particle responds to the forces generated by other charged particles. As everyone knows, like charges repel and unlike charges attract.

The electric force between two charged particles is given by

$$
F_{e}=k \frac{q_{1} q_{2}}{r^{2}}
$$

Here $r$ is the distance between the two charges $q_{1}$ and $q_{2}$, and $k$ is a constant that depends on the units being used for charge and distance in the expression. When many charges are involved, or the charges are distributed in space in complicated ways, using the simple force expression above is too difficult if one wants to find the totality of forces at work. Instead we turn to an electric "potential" model.

The electric potential is easily understood by thinking about how gravity works over a hilly landscape, as illustrated in the cartoon shown in Figure 9. The ball feels a force pulling it downhill because the potential energy is lower there. Rolling down, the ball accelerates as it coverts its potential energy into the energy of motion.

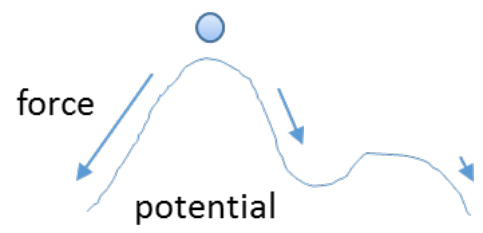

Figure 9. A cartoon showing how the force of gravity can be related to the difference in potential energy over a hilly landscape.

The same analogy holds for charged particles. There is an electric potential energy similar to the potential energy of gravity, and that potential is generated by all the electric charges that are near enough to a given charge to be felt. For a complicated problem, it is more practical to calculate the electric potential from a source distribution of charges, and then calculate the electric force at given point from the slope in the electric potential there, than to calculate the forces directly. Mathematical details can be found in any standard textbook on electrodynamics. ${ }^{2}$

Using the electric field analogy, we calculate the aversion force, given a discovery likelihood landscape instead of a charge landscape, calculating the potential field and then evaluating the field of aversion force of the same landscape. In the examples that follow, we evaluate the field given the perceived discovery risk for the second, less averse infiltrator (Figure 8).

\footnotetext{
2 Jackson, John David, Classical Electrodynamics, $3^{\text {rd }}$ Edition, Wiley 1998.
} 


\section{The Discovery Risk Perception Field}

In the ordinary course of reasoning, an adversary would not be inclined to distinguish between probabilities as numerical values on a continuum, but more likely to think in terms of discrete concepts described in words. For example, an adversary would not be very averse to being in a location where discovery is "unlikely," but, would strongly avoid places where discovery is "very likely."

To make the transition from probabilities expressed as numbers, to concepts described in language, we first consider what is already known about how humans talk about probabilities. The first formal consideration of this question was given by the intelligence analyst Sherman Kent in his research on "Words of Estimative Probability." 3,4 Since then, a number of authors have performed similar research. Table 1 is based on the work of Halliwell and Shen adapted for this demonstration. ${ }^{5}$

Table 1. Linguistic Terms for Probability Ranges

\begin{tabular}{|l|l|}
\hline Probability Range & Linguistic Category \\
\hline$<0.3$ & Unlikely \\
\hline 0.3 to 0.6 & Even Odds \\
\hline 0.6 to 0.8 & Likely \\
\hline$>0.8$ & Very Likely \\
\hline
\end{tabular}

This gives a correspondence between numerical values of probability and an ordinal set of labeled categories. However, it does not suggest anything about how strongly an adversary would respond to discovery probability categories expressed in these terms. For this we need to consult research on how humans perceive stimulus. Generally speaking, there is a logarithmic relationship between stimulus and perception. That's why the decibel system is used to describe how humans discern sounds based on its intensity. For demonstration purposes we consider a power-law relationship known as Stevens's Power Law. ${ }^{6}$

$$
\varphi(I)=k I^{n}
$$

Here $\varphi$ is a function of the perception of the stimulus intensity $I$ which, according to the law, is proportional to $I$ raised to the $n^{\text {th }}$ power. Since we are only conducting a demonstration, we choose an ad hoc scaling that we expect to give strong contrast across the range of linguistic categories. This scaling is shown in Table 2. Having each category differ by an order of magnitude from the prior one ensures that the adversary in our demonstration will strongly discriminate between one category and the next. The values in the table are very close to Stevens's Power Law for the case in which $I\left(p_{c}\right)=$ $p_{c} / p_{\text {even }}$, with $p_{c}$ being a given probability category and $p_{\text {even }}$ being the "Even Odds" category, and setting $k=1$ and $n=7.75$. For comparison, the exponent for stimulation of the finger by electric shock is $n=3.5$.

\footnotetext{
${ }^{3}$ Sherman Kent and the Profession of Intelligence Analysis, Center for the Study of Intelligence, Central Intelligence Agency, November 2002, p. 55.

${ }^{4}$ Words of Estimative Probability - Wikipedia, https://en.wikipedia.org/wiki/Words_of_estimative_probability

5 Joe Halliwell and Qiang Shen, "Linguistic probabilities: theory and application", Soft Computing, January 2009, Volume 13, Issue 2, pp 169-183.

${ }^{6}$ Stevens's Power Law - Wikipedia, https://en.wikipedia.org/wiki/Stevens\%27s_power_law.
} 
The necessity for categorizing and scaling the discovery likelihood field can be seen in the following examples. When we use only the bare discovery probability to calculate the aversion force field in the present case we obtain the field in Figure 10. Note that in region (a) the electric field points toward the low aversion portions of the terrain, while in region (b) the field seems not to see the border fence. This occurs because probabilities difference between the probability near the fence ( 0.6$)$, and in the nearby yellow regions ( 0.5$)$, is not very great on a linear scale.

Table 2. Probability Ranges, Linguistic Categories, and Scaled Risk Perception Values

\begin{tabular}{|l|l|l|}
\hline Probability Range & Linguistic Category & Risk Perception \\
\hline$<0.3$ & Unlikely & 0 \\
\hline 0.3 to 0.6 & Even Odds & 1 \\
\hline 0.6 to 0.8 & Likely & 10 \\
\hline$>0.8$ & Very Likely & 100 \\
\hline
\end{tabular}

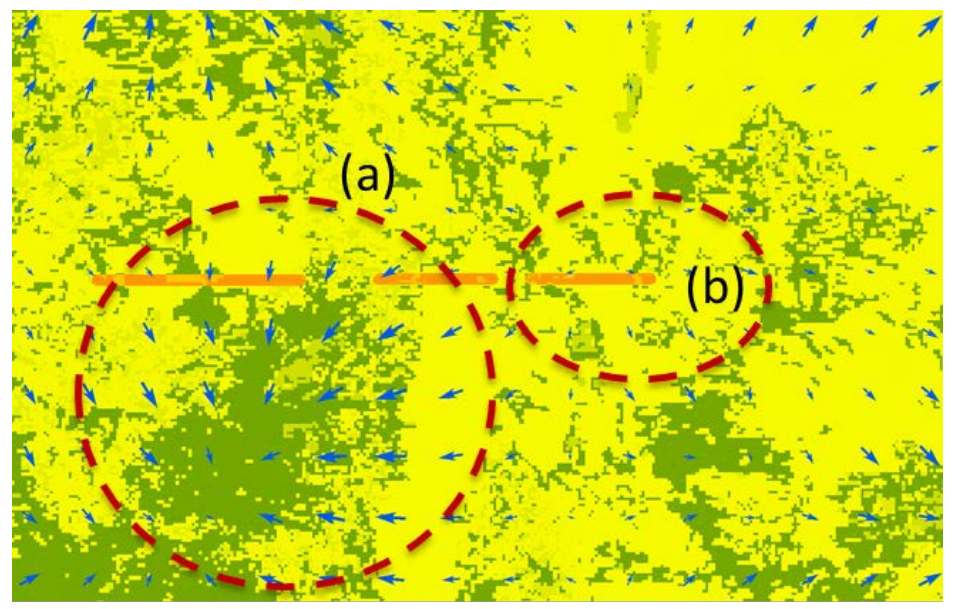

Figure 10. Resulting electric field vectors derived from the bare discovery likelihood field. Note that in region (a) the electric field points toward the low aversion portions of the terrain, while in region (b) the field seems not to see the border fence.

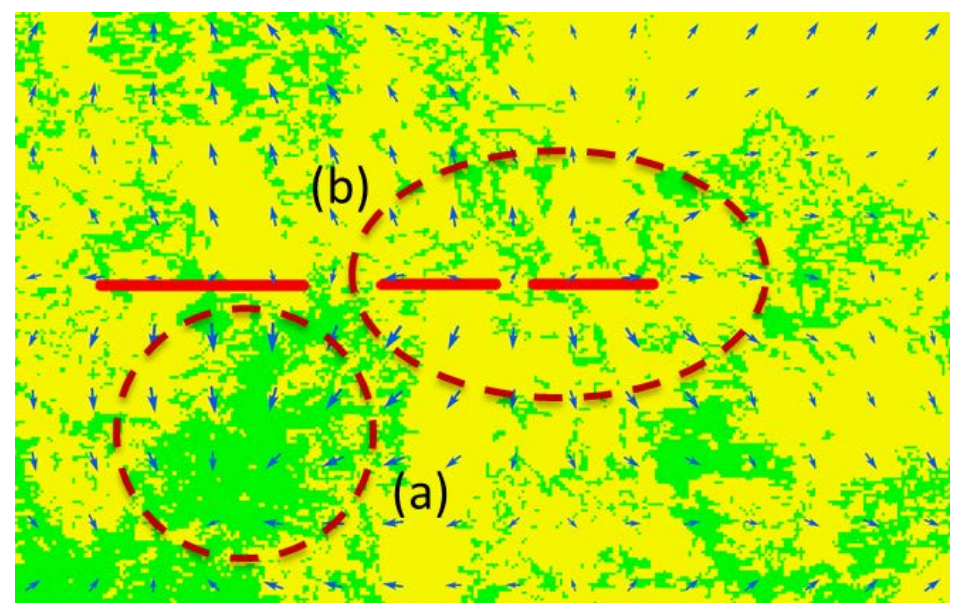

Figure 11. Resulting aversion field obtained by first scaling the discovery likelihood. In region (a) there are strong fields pointing toward favorable terrain, and in region (b) strong fields also point away from the border fence. 
We compare this with the results obtained by scaling the discovery likelihood field according to Table 2 . These are shown in Figure 11 where we can see that in region (a), there are strong fields pointing toward favorable terrain, and in region (b) strong fields also pointing away from the border fence. This result is owing to the fact that the perceived risk very near the border fence, and the risk associated with the nearby terrain, differ by an order of magnitude.

The complete sequence of steps in calculating the aversion force field is shown in Figure 12.
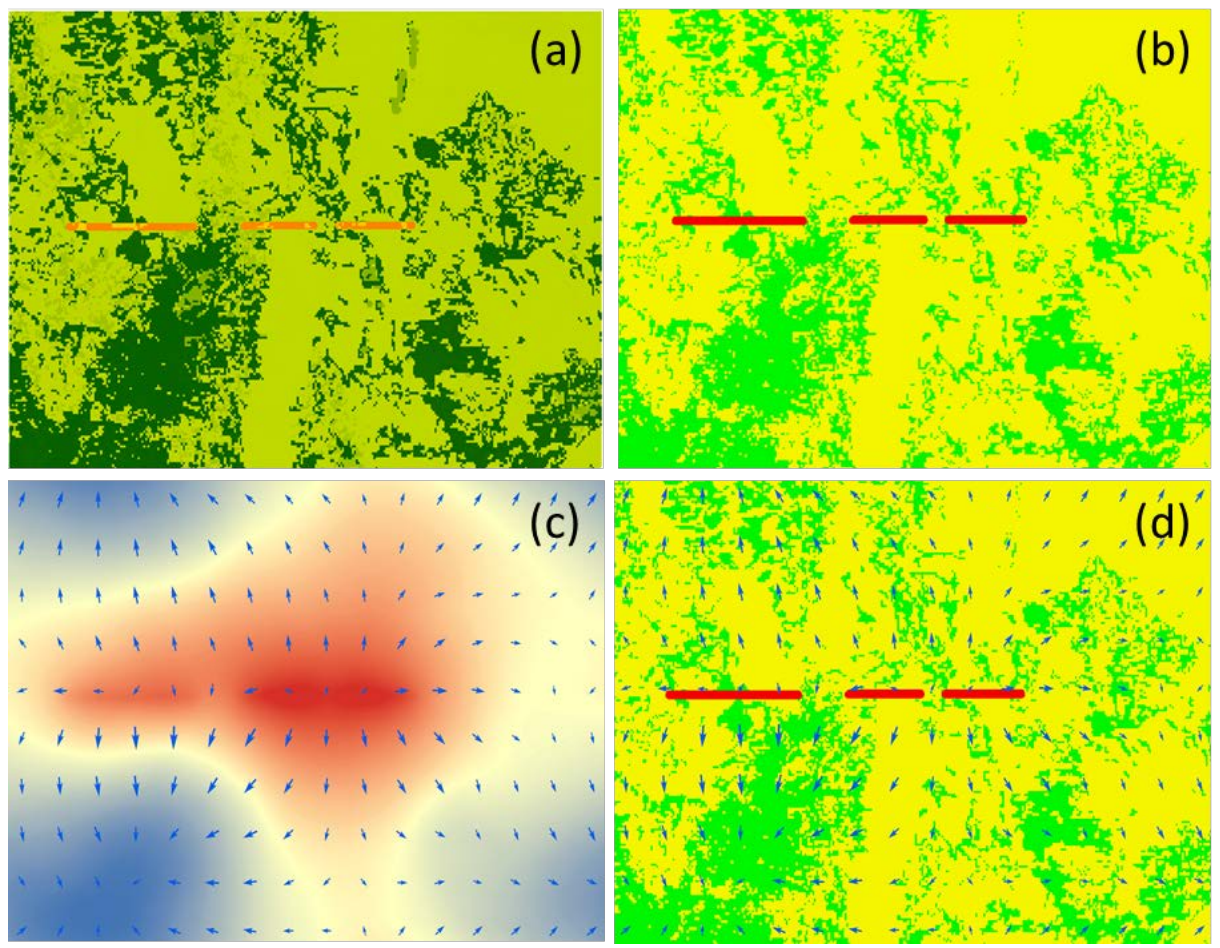

Figure 12. The steps in calculating the aversion force field: (a) discovery likelihood field, (b) risk perception field, (c) potential with aversion force field superimposed, (d) risk perception and aversion force superimposed.

\section{The Agent-Based Adversary Model}

The agent-based pathway model moves virtual adversaries in the aversive force field. There are two main influences on an adversary's motion:

1. An intention directing the adversary from their current location towards a goal destination

2. Deflection of the adversary from their intended direction by aversive forces

Given a starting location, a goal destination and intended speed, and an initial direction and speed, the agent-based model moves the adversary in discrete steps along the simulation landscape until they either reach their goal destination or leave the boundaries of the simulation. It's the interplay of the two main influences, intention and aversion, that ultimately determine the path that an adversary will follow in our model. These two main influences are also formulated using a physics analogy, namely that objects move under the influence of forces.

Figure 13 illustrates the interplay of these forces. The aversion force has already been described as a feature of the aversion field derived from the field of perceived discovery likelihood generated by the Bayesian network model. The intention force is new. 


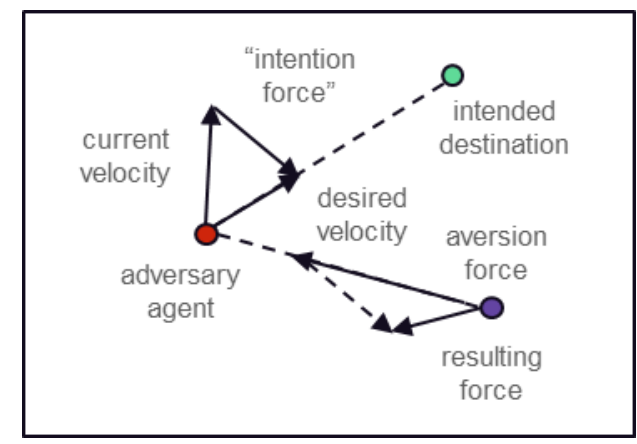

Figure 13. The interplay of forces in the agent-based model of adversary motion.

The idea is a simple one in physics. If an object is otherwise still or moving with a constant velocity, and it begins to move or changes direction, it is because it is under the influence of a force. A force is defined as the acceleration or change in velocity of an object proportional to its mass.

In our model, the adversary wants to be moving in a certain direction at a certain speed in order to reach their destination. If they are currently moving with a different direction and speed, then they must apply an acceleration to correct course. We call that the "intention acceleration."

$$
\vec{a}_{i}(t)=\frac{\left(\overrightarrow{v_{0}}(t)-\vec{v}(t)\right)}{\tau}
$$

Here $\overrightarrow{v_{0}}$ is the intended velocity (direction and speed), $\vec{v}(t)$ is the current velocity, and $\tau$ is a nominal reaction time during which the acceleration would take place. The formulation is attributed to Helbing and Molnar who successfully used this physics analogy in simulations of crowd evacuations.

The acceleration due to aversion is proportional to the aversion field $\vec{E}_{a}(x(t))$ calculated above, i.e.

$$
\vec{a}_{a}(x, t)=f \vec{E}_{a}(x(t))
$$

Here, the proportionality constant $f$ is simply called the aversion factor. The virtual adversary moves under the influence of the combined acceleration

$$
\vec{a}(x, t)=\overrightarrow{a_{\imath}}(t)+\overrightarrow{a_{a}}(x, t)
$$

At each step in time $\delta t$ their position changes according to

$$
\vec{v}(t+\delta t)=\vec{v}(t)+\vec{a}(x, t) \delta t
$$

Thus, for a given, calculated aversion field, and an adversary placed at a certain location with an intended destination and speed, these equations are evaluated at every time step producing a path for each simulated infiltrator.

Once the aversion field is generated, the behavior of individual agents can be further refined by means of the aversion factor. Following the electric field analogy, the $f$ factor is analogous to the charge-tomass ratio $q / m$ of a charged particle. For charged particles the parameters $q$ and $m$ have specific roles in influencing the trajectory of a particle in an electric field. The charge in the numerator determines the degree of force on a particle for a given field strength. Therefore, increasing $q$ directly increases the strength of the force. In the denominator, $m$ represents the degree of inertia in the response of a charged particle to a given force. Thus the larger $m$, the more resistant the particle is to changing 
direction. Making these comparisons we can then interpret $f$ as a ratio in which the numerator represents the degree of the aversion experienced by the adversary, and the denominator indicates the strength of the adversary to continue their present direction and speed in spite of the aversion they feel. With this in mind we can say that

- $f>1 \Longrightarrow$ the adversary is more fearful than determined

- $f=1 \Rightarrow$ the adversary is equally as fearful as determined

- $f<1 \Longrightarrow$ the adversary is more determined than fearful

In terms of the pathways traced out by virtual adversaries, we can expect that increasing $f$ will always cause the adversary to deflect more sharply from a given deterrence measure in the landscape than when $f$ was smaller.

To demonstrate the computation of pathways, we started ten agents representing infiltrators of the second type at equally spaced points on the bottom boundary of the test region (see Figure 8), with initial direction northward and preferred speed of $5 \mathrm{~km} / \mathrm{hr}$. We set a common goal destination for all agents as the center point of the top boundary. The time step was a quarter of an hour.

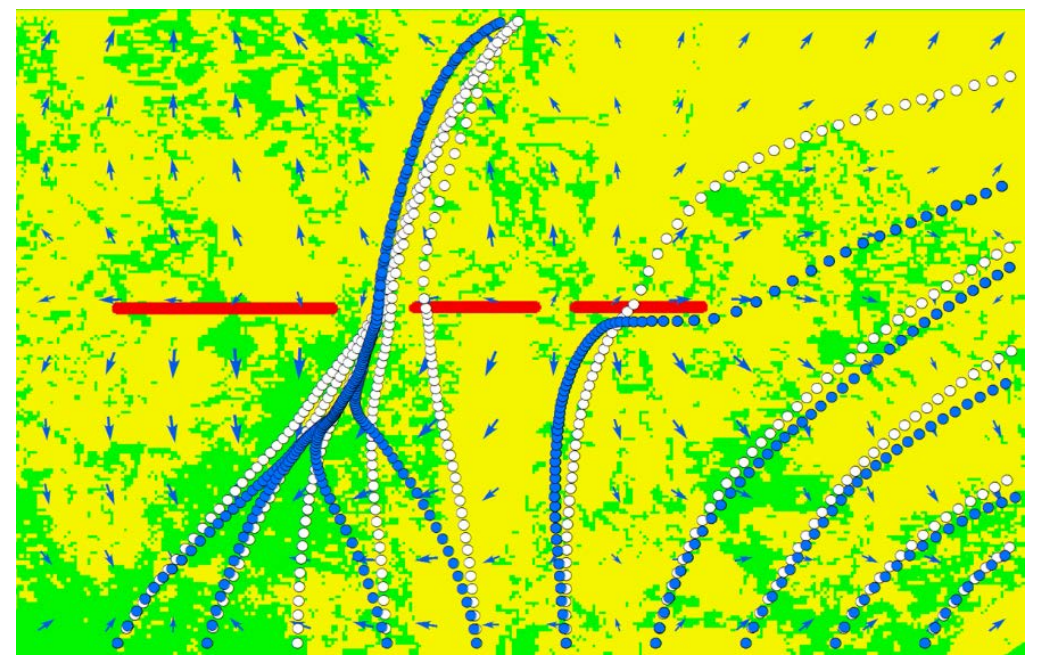

Figure 14. The pathways of ten virtual adversaries starting from locations equally spaced along the southern boundary of the test region each having as their destination the center of the northern boundary. White pathways: $f<1$; blue pathways $f>1$.

Note that agents having $f<1$ (white paths) are less likely to be deflected by the aversion force than their counterparts with $f>1$ (blue paths). This is especially evident near the barrier on the right where one of the blue paths is turned aside completely, whereas their counterpart on the white path is determined to go through. ${ }^{7}$

Path computations such as these can be used to estimate the probability of encounter by computing a large ensemble of pathways. By interpreting the pathways as an instantaneous snapshot of a continuous stream of agents (for example, introduced in the simulation at times $\delta t$ apart) the discrete locations in

\footnotetext{
${ }^{7}$ An adversary path that goes through a barrier would, in a more refined version of the model, revert to a submodel in the vicinity of the barrier to determine the behavior at that point. For example, if the barrier is not permeable to foot traffic, the model might apply a change in direction and speed in accordance with a particular rule such as reflection (the agent recoils from the barrier at an angle), or absorption (the agent is captured and removed from the simulation). If it is permeable it could simply retard the velocity and allow the agent to pass.
} 
the paths can be binned into cells of a geospatial grid and counted, thus providing an estimate of the frequency with which agents may be found in a given cell. This is the prior probability that a test subject (e.g., a Blue Force agent) in the same cell would encounter an adversary. The probability of encounter depends on how many such test subjects are present in the same cell. That is, the probability of encounter is the product of two probability density functions: $p_{A}(\vec{x}) p_{B}(\vec{x}) \delta A(\vec{x})$, where $p_{A}(\vec{x})$ is the probability density for adversaries, $p_{B}(\vec{x})$ is the probability density for some other type of agent (e.g., Blue Force agents), and $\delta A(\vec{x})$ is the area of the cell at the given location.

As an illustration, we have estimated the count of adversary agents $N_{A}(\vec{x})$ on a grid of cells superimposed on the test region. The paths of 1000 virtual adversaries were computed starting, as in the prior example, at equally spaced locations along the southern boundary, and having the same destination and starting conditions. The aversion factor for all paths was $f=4$, the same value that was used for fearful agents following the blue paths in Figure 14. The same time step of a quarter hour was also used.

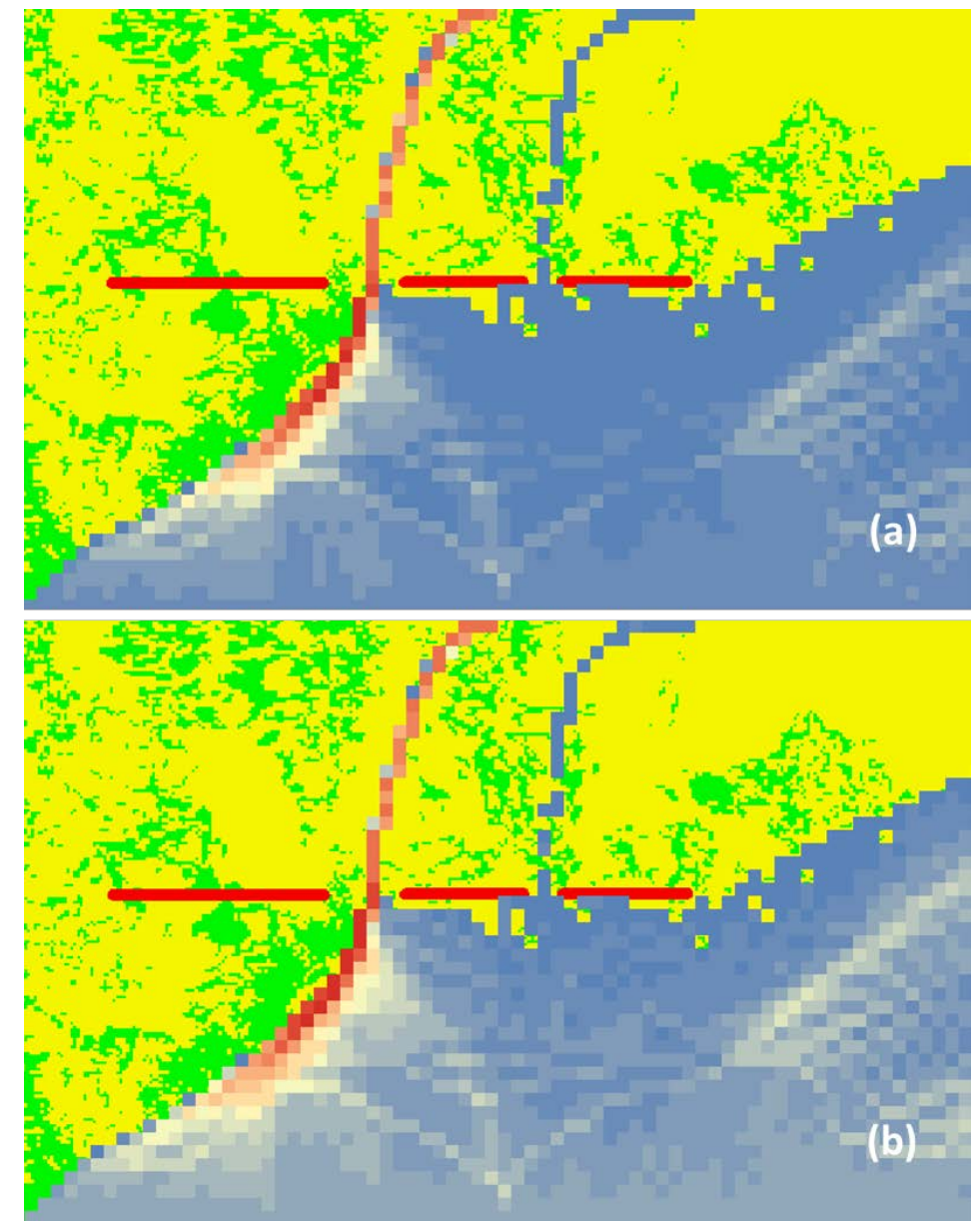

Figure 15. Adversary agent cell counts from a simulation involving 1000 agent pathways: (a) $N_{A}$, (b) $\log _{10}\left(N_{A}\right)$.

The results are shown in Figure 15. The upper figure (a) shows the number of counts $N_{A}$ and the lower figure (b) shows the log of the counts. We can see from both figures that one of the interesting features that emerges is a corridor for a likely adversary encounter along paths from the west and central portion of the southern boundary that merge into the predominantly red path segment to the southwest of the 
most westerly gap in the border fence and pass through it. An interesting feature of the log-scale plot is that certain statistically significant pathways can be seen. ${ }^{8}$ There are several from the southwest and two from the center that merge into the red segment south of the eastern gap in the fence. There is also a predominant pathway that emerges from the center of the southern boundary and then leaves the test region near the center of the eastern boundary, these adversaries having been unable to traverse the region successfully to their destination.

\section{Conclusions and Further Considerations}

We have described work performed to prove the feasibility of a novel modeling concept. The approach uses Bayesian networks and physics analogies to anticipate where infiltrators and smugglers that choose unconventional travel routes to evade discovery might be encountered. The first phase of the project has been a success. We believe the concept has the potential to contribute substantially to the ability of DHS and other federal agencies to solve difficult problems of this kind, and that the methods demonstrated in the first phase of the project are easily transferable to other contexts.

Maritime applications should be possible. Water is also open territory. The main difference in maritime applications is the lack of fixed barriers, which makes the problem somewhat more challenging. However, reasoning models that include attraction or aversion to specific landfall sites, along with information about resource constraints and the likelihood of patrols should help to narrow adversary choices. A similar approach could be used for non-commercial air travel.

We think that the idea could be applied, without much difficulty, to the problem of adversaries who choose to tunnel under borders or to avoid defensive forces to reach their objective. The geographic distribution of geologic features strongly influences where and how tunnels are built. Adding a subsurface layer of geologic information is therefore a straightforward extension. The subsurface layer can be coupled to the surface layer, along with its associated aversion field, to implicate locations where an adversary may feel relatively secure when entering or exiting a tunnel.

Application to air travel can be thought of in much the same way as the tunneling problem. Adversaries must travel over the surface to get to an airport. Once in the air, their routes are determined. However, their choices of source and destination airports are an integral part of their surface travel routes, and surface travel is affected by various aversive influences as we have demonstrated.

These considerations suggest that a natural extension of the concept would be to implement a multimodal, layered transportation architecture. The envisioned architecture would be made up of various surface layers to include rail, road, maritime, and open terrain. The architecture would also encompass air travel, as well as a geological subsurface layer. An interaction network would connect these layers. The interaction network would be based on an expanded reasoning model combining Bayesian and rulebased methods, as well as optimization techniques to determine where an adversary is most likely to transition from one layer to the next.

If it were undertaken to restart the project, we estimate, excluding administrative delays, that it would take approximately one month to reengage, depending on the availability of key technical personnel. How long the concept could remain shelved and effectively be restarted is more difficult to estimate. At

\footnotetext{
${ }^{8}$ These statistically suggestive pathways do not necessarily imply that there is any one pathway that resembles it. These are, strictly speaking, an aggregate effect of many pathways.
} 
the time of this report there are two key technical contributors with special expertise gained from their involvement in the project. It would remain straightforward to restart the project unless one of these key contributors were to leave the laboratory or become unavailable for other reasons. 